\title{
Influence of pattern coating thickness on porosityand mechanical properties of lost foam casting of Al-Si (LM6) alloy
}

\begin{abstract}
The combination of Aluminum alloy with lost foam casting (LFC) process is best applied in automotive industry to replace steel components in order to achieve light weight components for reducing fuel consumption and to protect the environment. The LFC process involves process parameters such as the degree of vacuum, foam degradation, expanded polystyrene (EPS) foam density, permeability of foam pattern coatings, pouring temperature, filling velocity, cooling rate, and pressure. The effect of polystyrene foam pattern coating thickness on the porosity and mechanical properties of Aluminum Al-Si LM6 alloy were evaluated experimentally. The coating thickness was controlled by slurry viscosity at range between $18 \mathrm{sec}$ to $20 \mathrm{sec}$ using Zahn viscosity cup No.5 and the foam pattern was coated up to fifth layer. Aluminum Al-Si (LM6) molten metal was poured into expandable mould and castings were examined to determine porosity distribution, mechanical properties and microscopic observation. Results from X-ray testing reveal the porosity distribution on Aluminum Al-Si LM6 castings is greater at thicker foam pattern coating sample. Meanwhile, the tensile strength of casting decreases when foam pattern coating thickness increases. Microscope observation portray the present of porosity on the casting which shows more gas defects present at thicker foam pattern coating sample. The source of porosity in LFC process is due to air entrainment or the entraining gases from polystyrene foam decomposition during pouring of molten metal. As a conclusion, mechanical strength has inverse relationship with porosity.
\end{abstract}

Keyword: Aluminium casting; Foam casting; Pattern coating; Porosity; Sand casting 